Technical Note

\title{
Design and Test of a New Inductive Force Sensor
}

\author{
Robert Bram Giesberts ${ }^{1}$ (D), Victor IJzebrand Sluiter ${ }^{1, *(1)}$ and Gijsbertus Jacob Verkerke ${ }^{1,2}$ \\ 1 Department of Biomechanical Engineering, University of Twente, Drienerlolaan 5, 7522 NB Enschede, \\ The Netherlands; r.b.giesberts@utwente.nl (R.B.G.); g.j.verkerke@gmail.com (G.J.V.) \\ 2 Department of Rehabilitation Medicine, University of Groningen, University Medical Center Groningen, \\ Hanzeplein 1, 9713 GZ Groningen, The Netherlands \\ * Correspondence: v.i.sluiter@utwente.nl; Tel.: +31-53-4896501
}

Received: 22 May 2018; Accepted: 28 June 2018; Published: 28 June 2018

\begin{abstract}
The currently accepted interval of weekly cast changes in the treatment of clubfeet seems unsubstantiated. A force sensor is needed to determine the adaptation rate of a clubfoot to establish what cast change interval would be most effective and efficient. We developed a force sensor based on the principle that the resonance frequency of an LC-tank changes when a metal target is brought in close proximity. A thin rubber ring between the LC-tank and the metal target transformed this proximity sensor into a force sensor. With a static load test and an incremental load test, the performance of the constructed force sensors was characterized. The custom-made sensor showed excellent sensitivity $\left(\left(1.7 \pm 0.8 \times 10^{5}\right)\right.$ counts $\left./ \mathrm{N}\right)$, resolution $((0.15 \pm 0.06) \mathrm{mN})$, and accuracy $((3.5 \pm 3.0) \%)$ for the application. The observed drift was $(2.1 \pm 0.7) \% / \log _{10}(\mathrm{~h})$, which is lower than other thin force sensors. Preliminary results of measurements in the treatment of Dupuytren fingers and clubfeet show good functioning for long-term force measurements.
\end{abstract}

Keywords: force sensor; induction; LDC; drift

\section{Introduction}

A clubfoot is a congenital deformity of the foot which is commonly treated with the Ponseti method [1]. In this treatment, in the first weeks after birth, the foot is manipulated towards a more corrected position and fixated with a plaster cast (Figure 1a). This weekly procedure is repeated several times until the deformity is fully corrected. At first the foot resists the newly imposed position by pressing against the rigid plaster cast, but over time the biological tissues inside the clubfoot adapt to the new position: the force against the plaster cast decreases (Figure 1b). The adaptation rate of a clubfoot can be determined by measuring the decrease of force between the cast and the clubfoot. This information is essential to improve the treatment method because it can determine when the next correction is due. This could be much faster than after one week.

To measure the adaptation rate of the clubfoot, a force sensor is needed with very specific requirements. The specific application that is described above requires a sensor that is thin enough to fit underneath the cast without causing any damage to the skin, accurate enough for a measurement over the period of one week and its power should be supplied by a small battery that would be safe in a portable device for a baby. The requirements used for the development of the force sensor described in this paper are summarized in Table 1.

Numerous force sensors with varying functional principles are commercially available, but, to the best of our knowledge, none of them are suitable for long-term precision measurements within a portable setting. Other sensors do not fit our requirements because they are either too bulky or experience too much drift. OEM load cells (e.g., Futek Inc., Irvine, CA, USA) are too large for our requirements, and it would be challenging to make a low-power amplifier with high resolution for load cells based on strain gauges. Force Sensitive Resistors (FSR, e.g., FlexiForce, Tekscan, Boston, 
MA, USA) are susceptible to drift [2-4]. OptoForce sensors (OptoForce, Budapest, Hungary) are elegant yet not thin enough to be placed below the plaster cast. TakkTile sensors (RightHand Robotics, Somerville, MA, USA) are a relatively good candidate, but we found that their measurement range is difficult to adjust and their accuracy over long-term measurements is unknown.

The aim of this paper is to describe the performance and accuracy of a new inductive force sensor for the application of long-term force measurements in a portable setting.

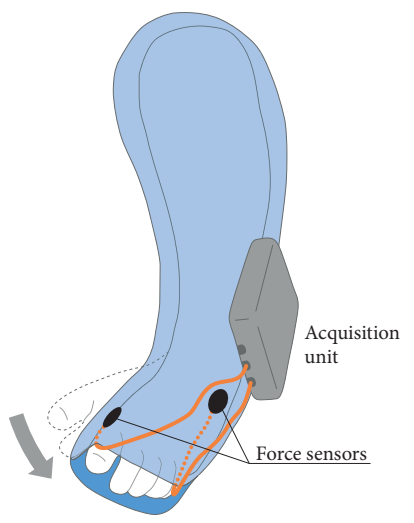

(a)

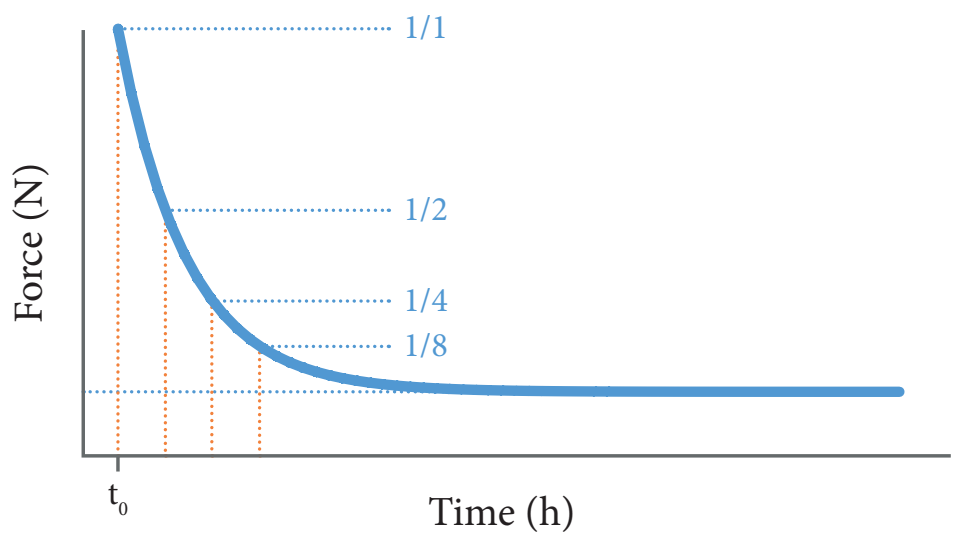

(b)

Figure 1. (a) Sensor location: The clubfoot is fixated in a corrected position with a plaster cast. (b) Expected plot: Initially the foot presses against the cast but over time this force decreases due to adaptation of the biological tissues.

Table 1. Sensor requirements. A small Lipo battery $(165 \mathrm{mAh})$ should be able to power the system for at least one week $(168 \mathrm{~h})$, hence the set requirement for the maximum power consumption.

\begin{tabular}{rlcl}
\hline Requirements & & Value & Unit \\
\hline Dimensions & $\max$ & $3 \times 10(\varnothing)$ & $\mathrm{mm}$ \\
Dynamic range & $\min$ & $0-10$ & $\mathrm{~N}$ \\
Resolution & $\max$ & 0.01 & $\mathrm{~N}$ \\
Time drift & $\max$ & 1 & $\% /$ day \\
Bandwidth & $\min$ & 0.1 & $\mathrm{~Hz}$ \\
Current consumption & $\max$ & 1.0 & $\mathrm{~mA} @ 3.7 \mathrm{~V}$ \\
\hline
\end{tabular}

\section{Sensor Design}

An inductive force sensor was developed based on the evaluation kit of the inductance to digital converter LDC1000 (Texas Instruments, Dallas, TX, USA) and its successor LDC1614 [5]. This integrated circuit is able to accurately determine the resonance frequency $\left(f_{\text {sensor }}\right)$ of an LC-tank. Its main output parameter is LHR_DATA, a digital value that is proportional to frequency, with 28 bits of measurement resolution. The resonance frequency is influenced by the distance of the coil to any metal target. In most applications, a non-ferromagnetic and conductive metal (e.g., aluminum) is used to modulate the inductance of the coil. Closer proximity of the target to the coil will increase eddy currents in the target, thereby decreasing the inductance of the coil. In close proximity, the relation between distance and resonating frequency can be linearized with small errors. The exact functioning and examples for sensor design can be found in the documentation provided by Texas Instruments e.g., [6,7].

To convert this proximity sensor into a force sensor, an elastic medium with a known stiffness was added between the coil and the target. Our initial approach consisted of a simple sandwich of these three components. Although this solution has been researched by others [8], and patented by others a.o., [9,10], it did not appear as a commercial product at the time of writing of this manuscript. Preliminary tests with this approach showed a high accuracy and sensitivity but mediocre long-term 
results, with time drift values as high as 50\%/day. Several tests were conducted to identify the cause for this drift. If the sensor was not loaded with any weight, no drift was observed. Creep of the rubber medium was rejected with a creep test under $50 \%$ compression for 14 days, which showed no deformation. Replacing the compressible medium by an equally thin sheet of (incompressible) glass did not resolve the drift. Neither did replacing the aluminum target for a polyethylene target. One of the few hypotheses remaining was plastic deformation of the PCB.

In the final design, a base-plate was added and the rubber medium was shaped into a "ring", enclosing the PCB (see Figures 2 and 3). With this approach, loading the sensor compresses the rubber ring around the PCB but prevents loading - and possibly deforming - the PCB. With the rubber ring, this solution is a compromise on the size ( $\varnothing 10 \mathrm{vs} . \varnothing 7 \mathrm{~mm}$ ). A DS1825 thermometer [11] was added to correct for the influence of temperature.

The terminology used for the sensor, the acquisition unit (AU), and their combination as a sensing and logging unit (SLU) throughout this paper are presented in Figure 2.

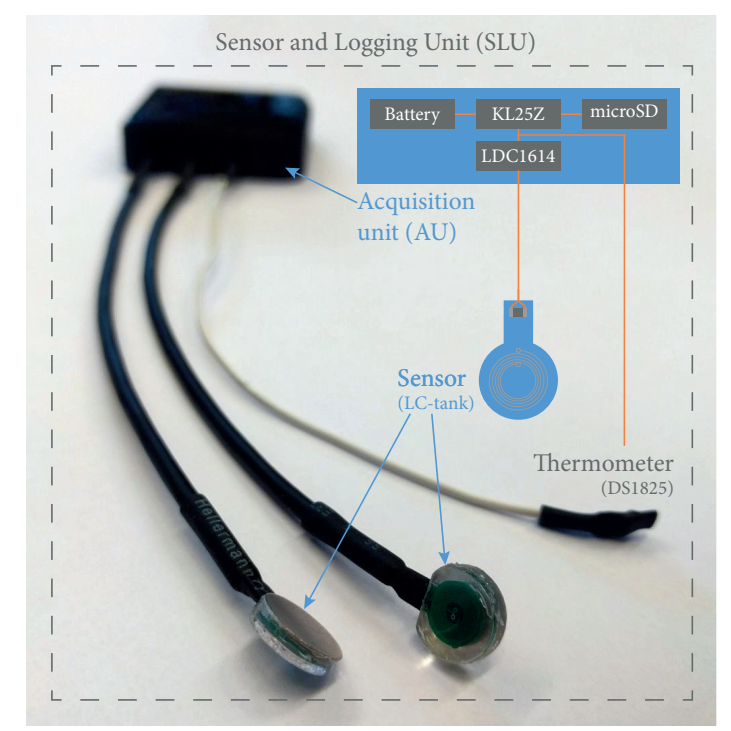

Figure 2. Two sensors and an acquisition unit (AU). The AU (top) allows the connection of two sensors (below) and a thermometer (right). Schematic representation of a sensor and logger to explain the distinction between sensor, AU, and the combination of the two: the sensing and logging unit (SLU).

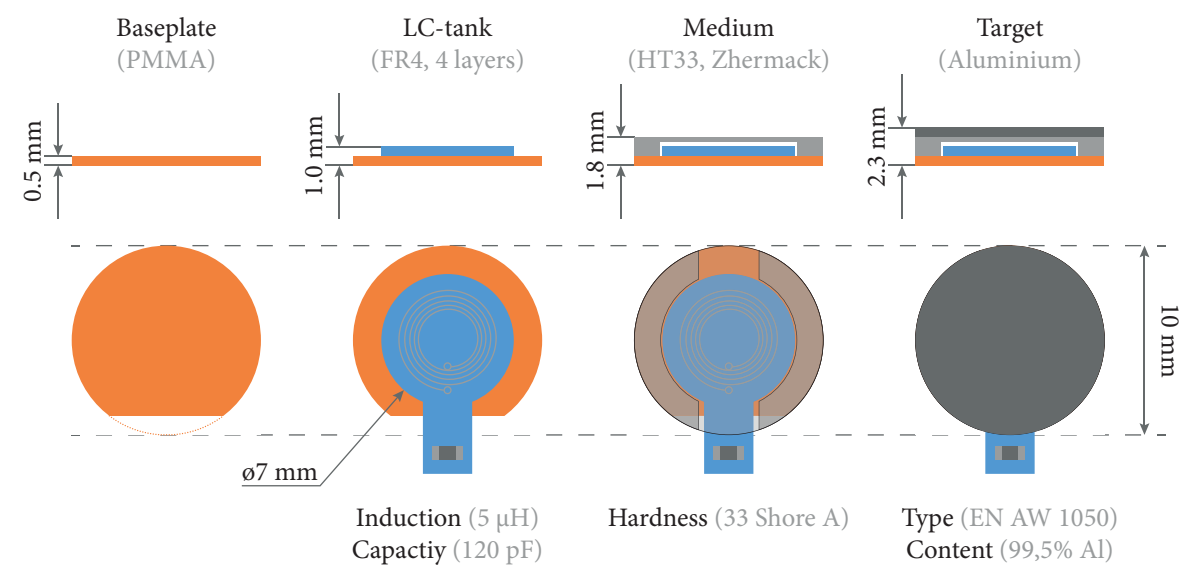

Figure 3. Sensor specifications. Front and top view of the sensor in four stages of the assembly process. The sensor is built out of four parts of which the technical specifications are given here. 


\section{Acquisition Unit}

The sensor was connected to a custom-made AU to log LHR_DATA over time. The AU was equipped with the LDC1614 [5] and a KL25Z microprocessor [12] that was programmed to write the collected data to a microSD memory card. The AU was powered with a small single cell Lipo battery (165 mAh).

Programming of the AU was optimized for high sensitivity, low power consumption, and little time drift. This means that for the internal settings of the LDC1614, CHx_RCOUNT was set to maximum (OXFFFF) for increased precision at the expense of bandwidth, SETTLECOUNT_CHx to 50 (to allow a sensor settling time of $t_{\text {settle }}=20 \mathrm{~ms}$ ), and CHx_DRIVE was automatically determined using the method described by Holubeva [13] and set to 23. The resulting sample rate was $18 \mathrm{~Hz}$. The used code is available online [14].

\section{Experimental Section}

\subsection{Data Collection}

To reduce energy consumption, the AU was programmed to write packages of 60 data samples at a time to the microSD card, rather than sample-per-sample. The thermometer was programmed to do one temperature measurement per package.

\subsection{Test Setup}

A calibration rig was fabricated which allowed us to hang known weights on the sensor. The applied load $(F$, in $\mathrm{N})$ compresses the sensor, thereby changing the oscillation frequency. LHR_DATA, proportional to this frequency, is measured and stored by the AU. None of the parts used in close proximity of the sensor were made of metal to prevent any interference with the LC-tank. A hanger of $127 \mathrm{~g}$ was used as a pre-load to which weights of $100 \mathrm{~g}$ each were attached (see Figure 4).

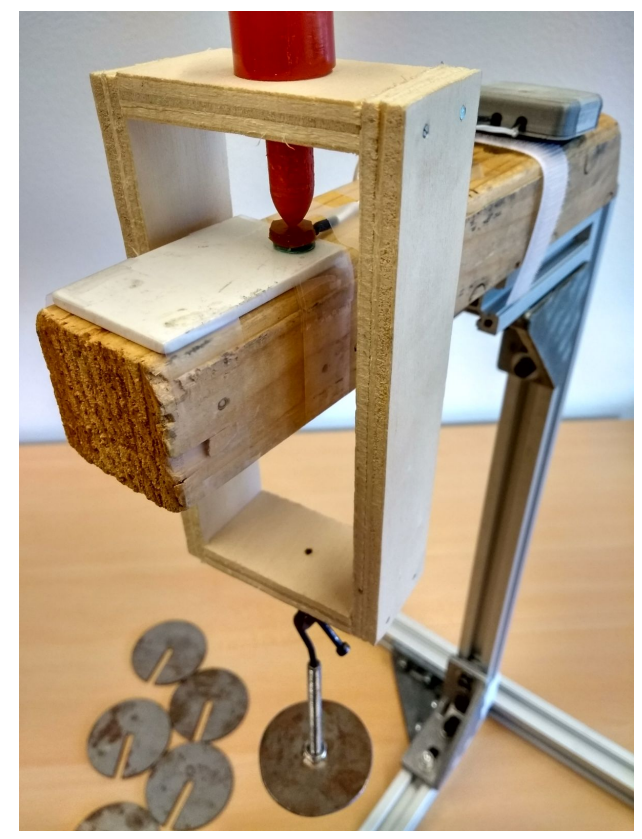

Figure 4. Calibration rig. Each sensor was placed in the calibration rig. Steel disks of $100 \mathrm{~g}$ each (bottom left) were placed on the hanger to apply a load.

\subsection{The Static Load Test}

A static load test was performed to carefully assess time drift of the SLU. The SLU was placed in the calibration rig with the pre-load in place. During the first $2 \mathrm{~h}$, the force sensor was not loaded, then 
a weight of $500 \mathrm{~g}$ was hung on the force sensor and left there for at least $12 \mathrm{~h}$ after which the load was removed. Measurements continued for another $2 \mathrm{~h}$.

The following characteristics were determined:

Noise was defined as the standard deviation of the output signal (LHR_DATA), filtered with a high-pass filter $(0.01 \mathrm{~Hz})$. Furthermore, the noise performance was characterized by measuring the Allan deviation [15] (p. 15) as a function of averaging time using a sensor that was not loaded for eight hours.

Temperature drift of the SLU was defined as the change in LHR_DATA per change of the room temperature (heating of the empty office used for our tests was controlled centrally and caused fluctuations in temperature). It was calculated as $\triangle$ LHR_DATA / $\Delta T$ over the period while the sensor was loaded and the input parameter $(F)$ was constant, in counts $/{ }^{\circ} \mathrm{C}$.

Time drift of the SLU was defined as the relative change of LHR_DATA over time, after a $30 \mathrm{~s}$ settling time. Two calculation methods were used:

- Drift was calculated as the gain of the best linear fit of the signal over logarithmic time, in $\% / \log (\mathrm{h})[16]$.

- Drift was calculated as the relative difference between the initial value and the final value after several specific times [3].

\subsection{The Incremental Load Test}

The SLU was loaded with incremental 30-second-long steps of $100 \mathrm{~g}$ each up to a maximum of $1 \mathrm{~kg}$. With this test, the following characteristics were determined:

Sensitivity of the SLU was defined as the change of the output signal per change of input parameter $\left(\triangle \mathrm{LHR} \_\right.$DATA $\left./ \Delta F\right)$ [17]. It was determined as the gain of a best linear fit in counts/N. Non-linearity was defined as the greatest deviation to this linear fit [17].

Accuracy of the SLU was defined as the greatest deviation to a polynomial fit that was used to allow conversion of LHR_DATA to force, as percentage of the full scale output.

Hysteresis of the SLU was defined as the maximum difference between the loading and unloading curves, as percentage of the full scale output.

\subsection{Data Processing}

The collected data were processed using Matlab R2017b. In post-processing, the LHR_DATA for the static load tests were corrected for an apparent start-up effect and for the influence of temperature changes using the identified temperature drift.

\section{Start-Up Effect}

In its intended application in force measurements in the treatment of clubfoot, the SLU uses a logging protocol of four $30 \mathrm{~s}$ periods per hour to preserve battery power. Every time the SLU is activated an initial start-up effect is observed, as shown in Figure 5. Seemingly independent of external influences, the output signal consistently decreases over time to reach a plateau after several minutes. This behavior was observed in all SLUs with almost identical parameters. For all sensors, this behavior was identified, quantified, and corrected. 


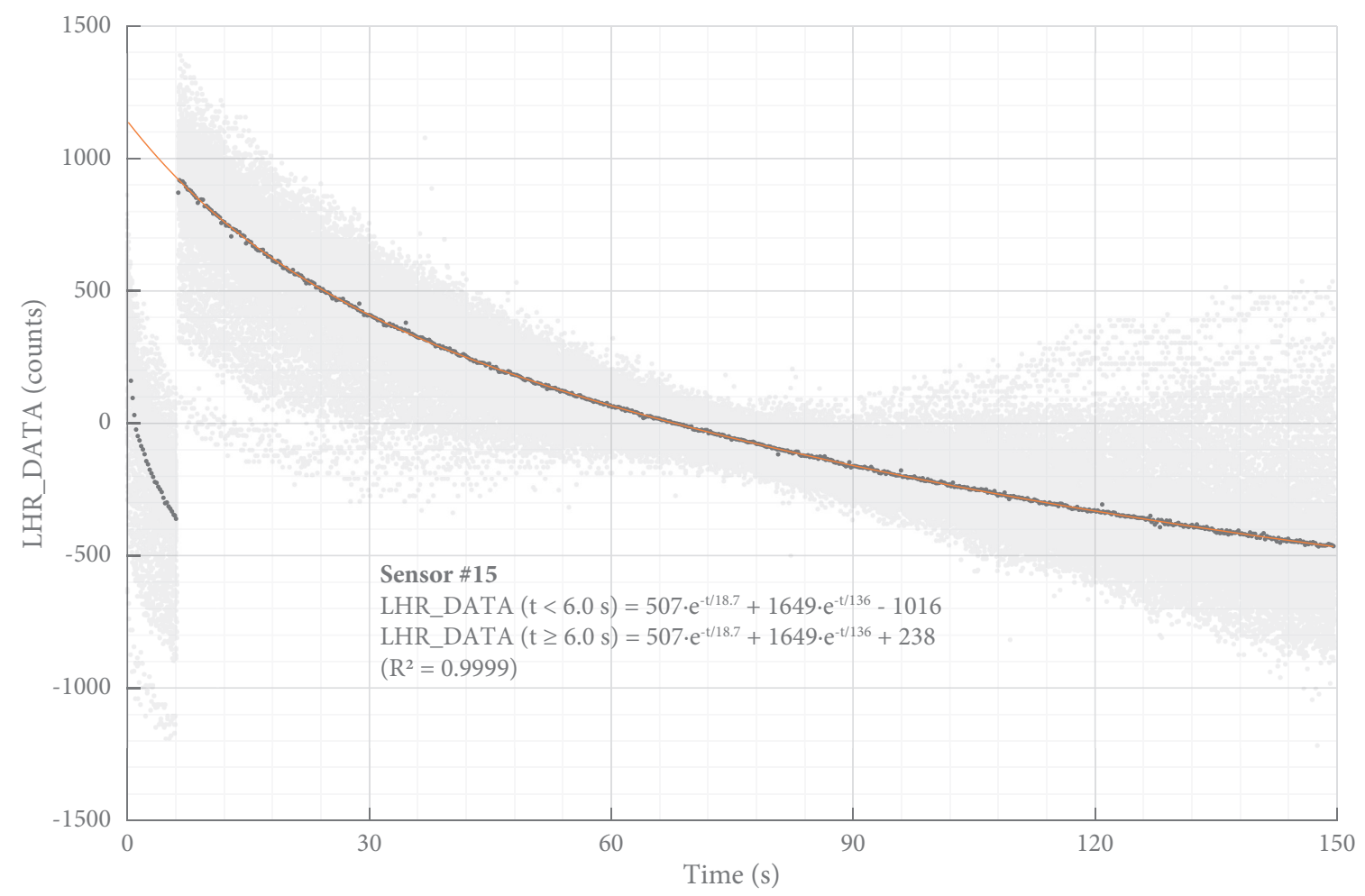

Figure 5. Start-up effect. The output signal gradually decreases every time the SLU is started. For this graph, the SLU was programmed to take ten $150 \mathrm{~s}$ measurements per hour, (gray). For all timestamps, the mean was calculated (dark gray), and least squares regression was used to find the best fit to a double exponential function (orange). The typical sensitivity (Table 2) can be used to convert LHR_DATA to a measure for force: 1500 counts approximate $0.01 \mathrm{~N}$.

\section{Results}

The static load test was performed with 9 sensors and the incremental load test with 13. Typical examples of the resulting data of both tests are presented in Figures 6 and 7, respectively. The performance of the inductive force sensors are summarized in Table 2 and described in more detail below.

Table 2. Sensor performance. All characteristics are presented as mean \pm standard deviation.

\begin{tabular}{lcc}
\hline & $\begin{array}{c}\text { Static Load Test } \\
(\boldsymbol{n}=\mathbf{9})\end{array}$ & $\begin{array}{c}\text { Incremental Load Test } \\
(\boldsymbol{n}=\mathbf{1 3})\end{array}$ \\
\hline Sensitivity $\left(10^{5}\right.$ counts/N) & $1.7 \pm 0.8$ & $1.3 \pm 0.6$ \\
Non-linearity $(\%)$ & & $12 \pm 10$ \\
Accuracy $(\%)$ & $5.8 \pm 1.4$ & $3.5 \pm 3.0$ \\
Hysteresis $(\%)$ & $1.2 \pm 0.4$ & \\
Noise $\left(10^{-3} \mathrm{~N}\right)$ & $0.15 \pm 0.06$ & \\
Effective Resolution $\left(10^{-3} \mathrm{~N}\right)$ & $2.1 \pm 0.7$ \\
Time drift $\left(\% / \log _{10}(\mathrm{~h})\right)$ & $5.0 \pm 1.4$ & \\
Time drift after $8 \mathrm{~h}(\%)$ & $-0.088 \pm 0.030$ & \\
Temperature drift $\left(\mathrm{N} /{ }^{\circ} \mathrm{C}\right)$ & & \\
\hline
\end{tabular}

The influence of temperature was $(-0.088 \pm 0.030) \mathrm{N} /{ }^{\circ} \mathrm{C}\left(\mathrm{R}^{2}=0.84\right)$. The results of a typical temperature analysis are shown in Figure 8.

The time drift of the SLU was determined to be $(2.1 \pm 0.7) \% / \log _{10}(\mathrm{~h})$. Figure 9 shows the observed drift during a typical incremental load measurement. 


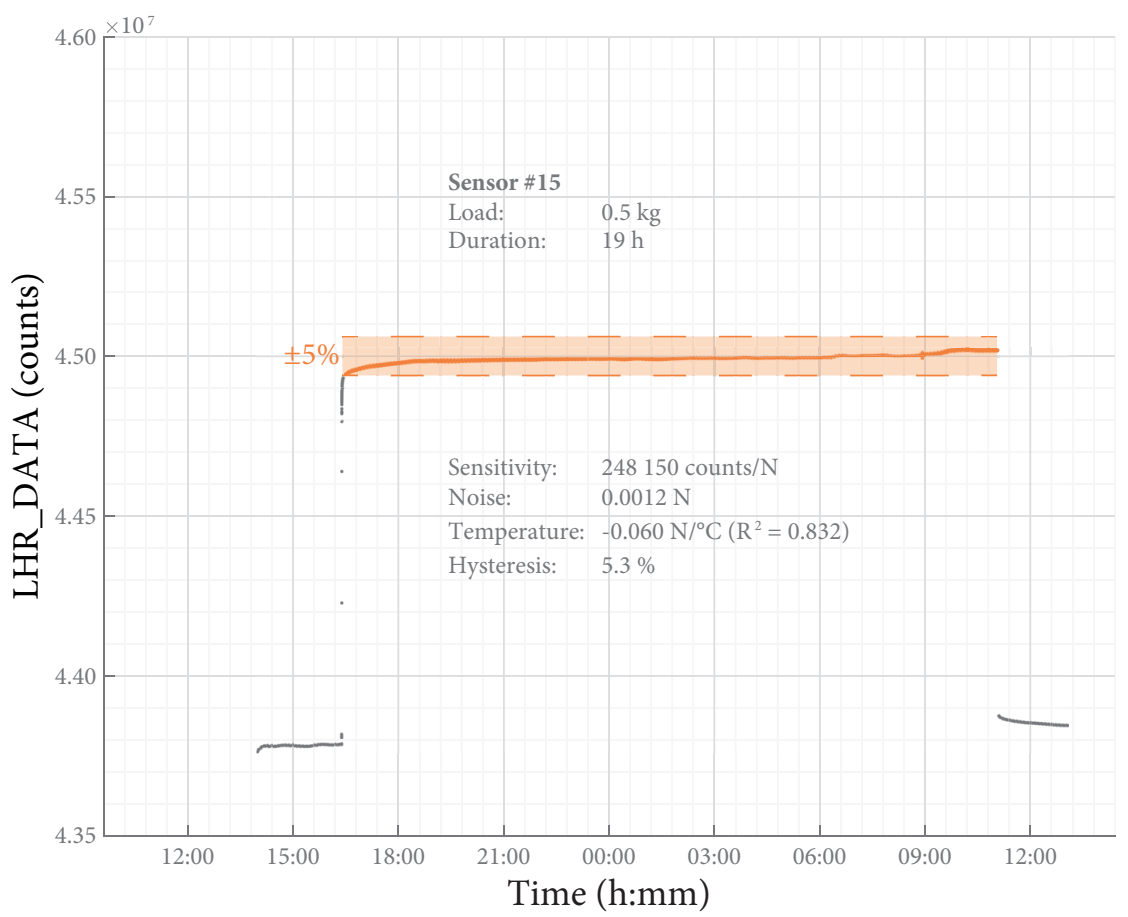

Figure 6. Static load test. The results of a typical static load test. The typical sensitivity (Table 2) can be used to convert LHR_DATA to a measure for force: 1500 counts approximate $0.01 \mathrm{~N}$.
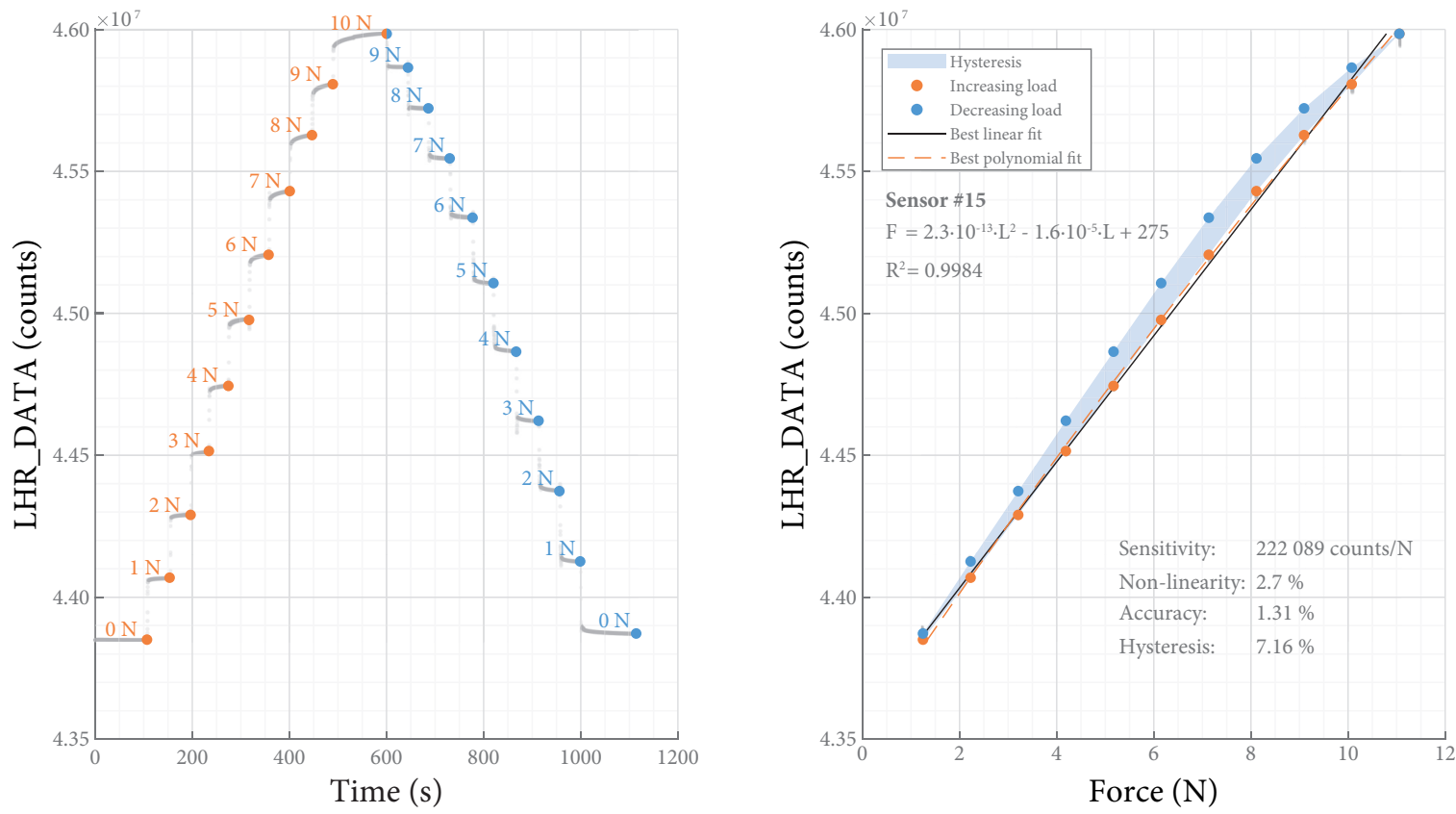

Figure 7. Incremental load test. The results of a typical incremental load test. 


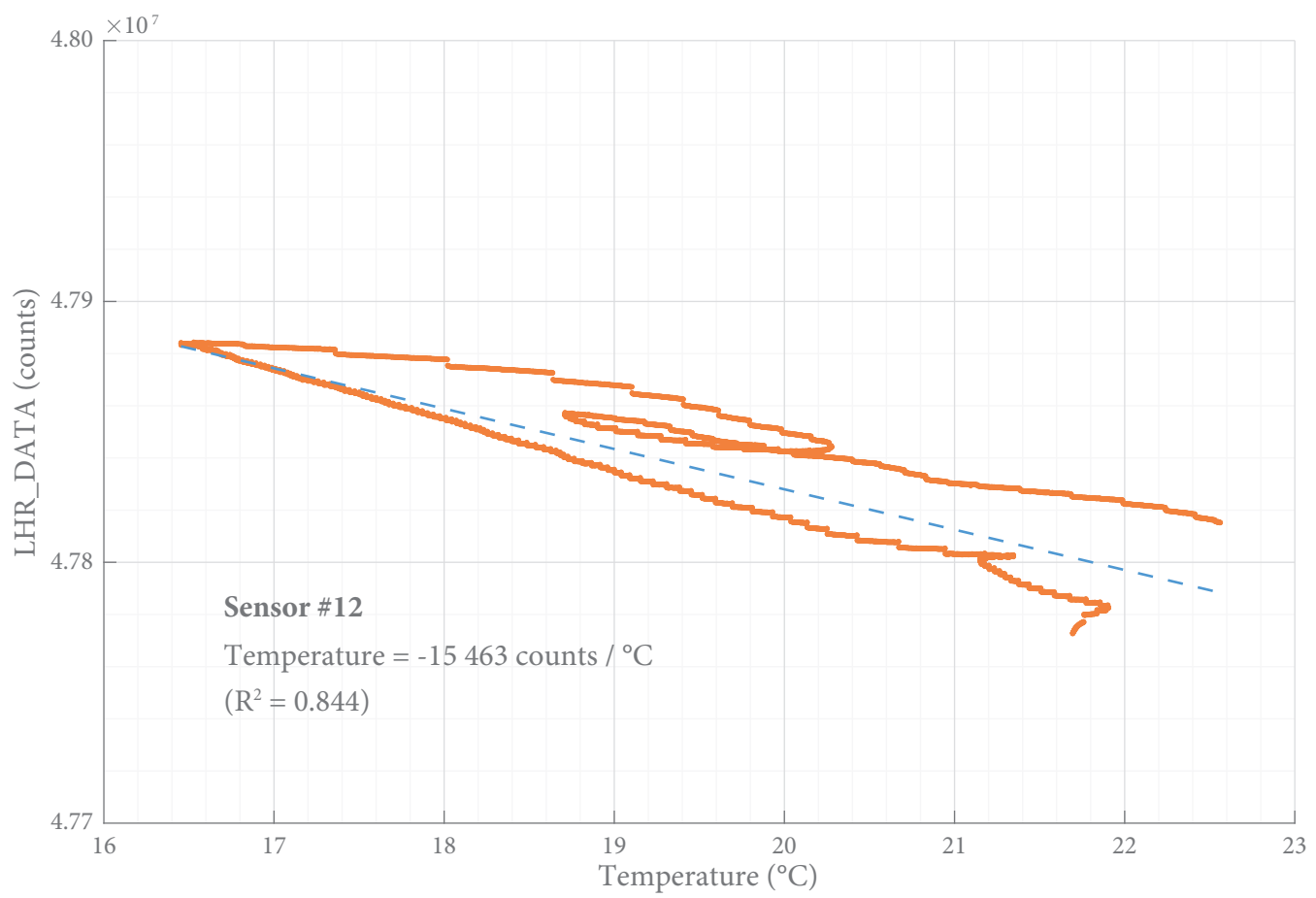

Figure 8. Temperature influence. About $85 \%$ of the changes in output signal can be explained by fluctuations in temperature. It is not clear if this temperature influence is independent of the observed time drift and the load applied. The typical sensitivity (Table 2) can be used to convert LHR_DATA to a measure for force: 1500 counts approximate $0.01 \mathrm{~N}$.

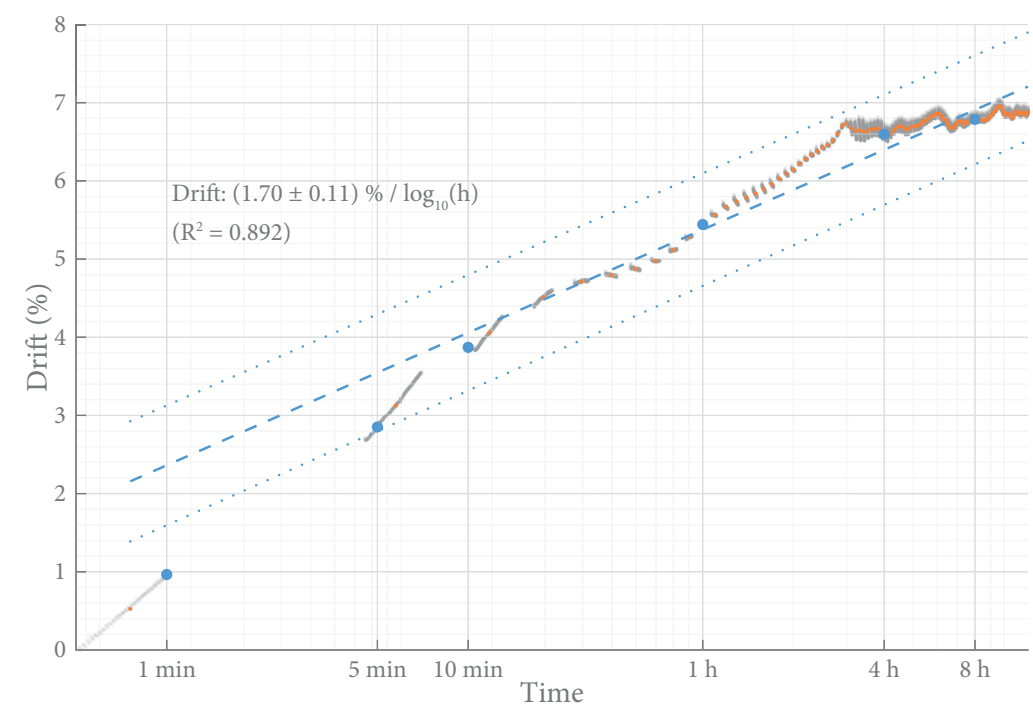

Figure 9. Time drift. The results of a typical static load test, during the loaded period, with time on the $x$-axis on a logarithmic scale. Orange dots represent the observed drift. A best linear fit and its $95 \%$ prediction interval is presented as a blue line. The blue dots represent the drift at specific times, as per Parmar et al.'s [3] method.

The noise characteristics are presented in the Allan deviation graph in Figure 10. It shows that the contribution of white noise is relatively small and that the sensor response is dominated by drift. 


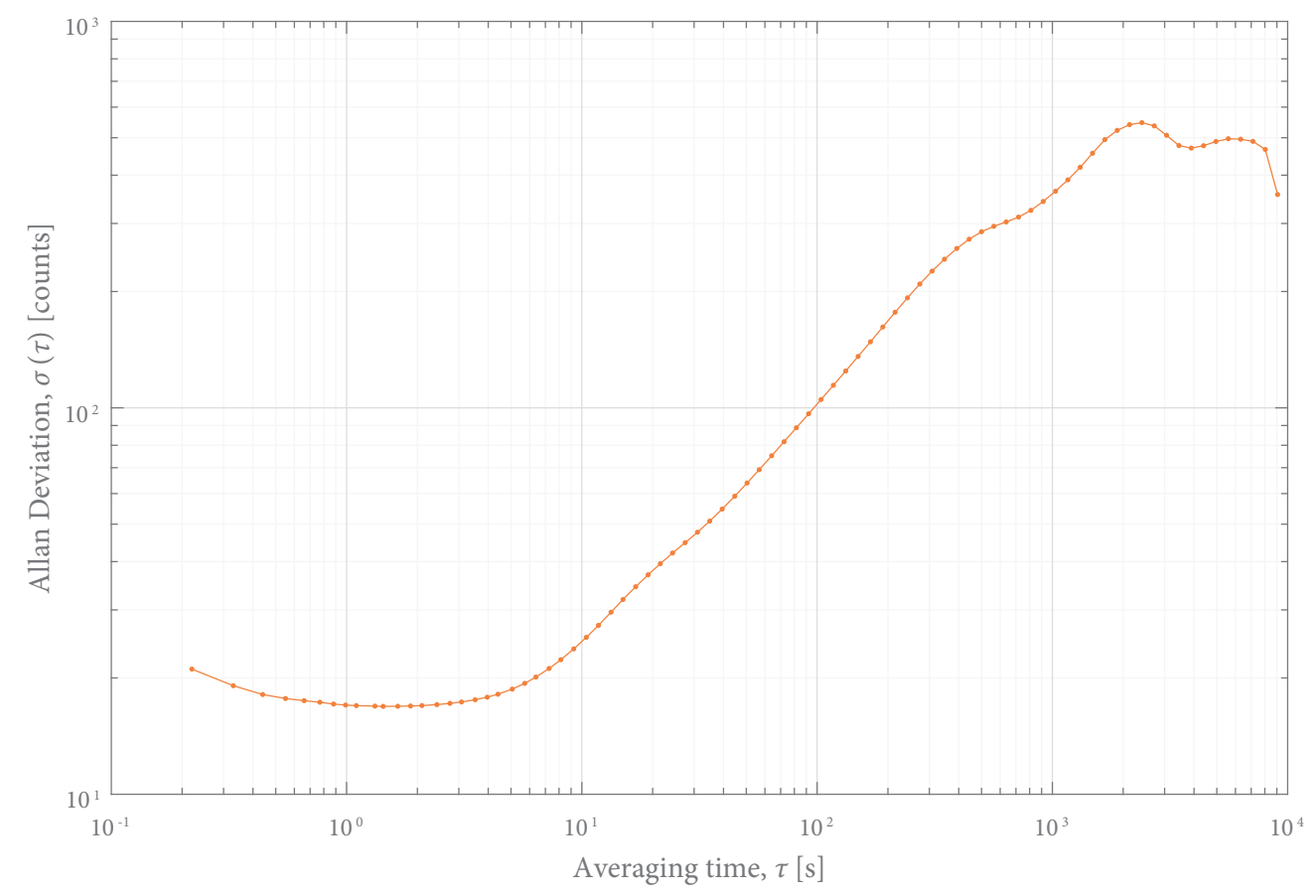

Figure 10. Allan deviation The brief decline at the onset of the curve indicates that the contribution of white noise is relatively small. The upward slope shows that the sensor response is dominated by drift. It can be concluded that the optimal averaging time is around $2 \mathrm{~s}$.

\section{Total Error}

The greatest sources of error are hysteresis (both ways, $\pm 2.5 \%)$ and drift $\left( \pm 2.1 \% / \log _{10}(\mathrm{~h})\right.$ ). When the output signal is corrected for the start-up effect and for the influence of temperature, the combined error is approximately $\pm 5 \%$ of the full scale output. Depending on the application, the actual error might be higher due to influences that were not present in the tests used in this study.

\section{Discussion}

\subsection{External Influences}

A negative linear relationship was observed between a change in temperature and the output signal. This means that, with an temperature increase, the output signal suggests a larger distance between the LC-tank and the aluminum target. Thermal expansion of the rubber medium might be a cause for the observed temperature drift.

Most of the fluctuations of the sensor signal under a constant load could be explained by changes in temperature $\left(\mathrm{R}^{2}>0.84\right)$ and the signal was corrected for this influence. However, some residual fluctuations could be observed-other than noise or drift—with an unknown source. These fluctuations might be caused by higher order effects of temperature or by any other external influence, but this was not studied extensively.

\subsection{Effective Resolution}

The LHR_DATA consists of 28-bit numbers, but the resolution of the full output range is closer to 23-24 bit. This is due to 20-22 count differences between possible sample values. This quantization fits the documentation of Oberhauser [18], which predicts an LDC resolution of $3 \mathrm{~Hz}$ (corresponding to $\sim 20$ counts in our reading) when an external oscillator of $40 \mathrm{MHz}$ is used. As the sensitivity is specified using LHR_DATA, the effective resolution is 20-22 times the reciprocal of the sensitivity. 
The sensors were calibrated over a range of $10 \mathrm{~N}$, which generated an output signal covering approximately $1 \%$ of the 28 -bit LDC range. This means that over the relevant force used, the sensor signal spans a 16-bit range. In practice, the sensor is able to detect changes in load in the range of tens of $\mathrm{mN}$.

\subsection{Drift}

Although drift is a well-known phenomenon, different methods exist for its quantification. Because of its apparent randomness, it can be difficult to give a clear figure for drift. It is presented as a percentage after a specific time $[3,4]$ or as a percentage per logarithmic hour $[16,19,20]$.

Reports on force sensors with similar designs do not present a characterization of drift [8] and did not allow for any meaningful comparison, so we have compared the observed drift in our sensor to that of commonly used force sensors. We chose Parmar et al.'s [3] definitions and methods, who evaluated several force sensitive resistors (FSRs) and applied $72.7 \mathrm{mmHg}$ to their sensors. After $8 \mathrm{~h}$, the signal had drifted with $10-25 \%$ compared to the value after an initial settling time of $26 \mathrm{~s}$. Using the same protocol Khodasevych et al. [4] found $0.4-24 \%$ drift after $8 \mathrm{~h}$ for more FSRs. In our static load test, a weight of $500 \mathrm{~g}$ was placed on the sensor with a $10 \mathrm{~mm}$ diameter, equal to $74.6 \mathrm{mmHg}$. After $8 \mathrm{~h}$, our sensors showed $5.0 \pm 1.4 \%$ drift.

The chosen settling time has a large influence on the figure for drift. Every sensor has some transient behavior before reaching its steady state, but the exact end of the transient behavior is hard to find and might be after several hours. The choice for a settling time of $26 \mathrm{~s}$ [3] or $30 \mathrm{~s}$ (our study) is arbitrary and creates a risk of bias. In most cases, drift is not purely linear but rather logarithmic, so the choice for a longer settling time generates a lower value for drift. Additionally, a longer settling time creates a higher reference value $\left(P_{i}\right.$ in Parmar et al. [3]), which in turn also lowers the value for drift.

Figure 7 shows an apparent time response (whether it be called response time or drift) after every change in load in the incremental load test. While other sensors might have a clear distinction between response time and drift, our sensor does not: these characteristics rather seem to overlap. Due to drift of the sensor signal, it is impossible to determine a stable "end" value without choosing an arbitrary time span. It is therefore also not possible to determine how long it takes to reach this value. With the drift present, any calculation of response time would be influenced by the choice for an arbitrary time span and is therefore omitted in this paper. Our choice for a settling time of $30 \mathrm{~s}$ is arbitrary and of pragmatic nature.

Any quantification of drift should be practical, and presenting it as a percentage per logarithmic time gives a better idea about the error that has to be taken into account when studying the sensor data. According to the datasheets of various FSRs $[19,20]$, their drift is $<5 \% / \log _{10}(\mathrm{~h})$. Using the same calculation method, our sensor shows a logarithmic drift of $(2.1 \pm 0.7) \% / \log _{10}(\mathrm{~h})$.

\subsection{Reflection}

For the specific application of long-term force measurements in the treatment of clubfoot, the performance of the developed force sensor fits most requirements. The sensor is thin enough to be placed underneath the cast without causing skin problems, and the AU is neither bulky nor heavy. The observed drift is better than for most FSRs $[3,19,20]$ but slightly higher than required. In our application, the force is expected to slowly decrease to a certain minimum level. In those measurements, the error caused by drift might be limited since the observed drift appears to be load-dependent.

The influence of temperature cannot be corrected perfectly and some residual error can be expected. However, the temperature underneath a plaster cast is not expected to fluctuate much and by using an external thermometer most of the temperature influence can be corrected in post-processing. Hysteresis cannot be corrected for since the actual load on the sensor is unknown and therefore the loading history cannot be compensated for.

All sensors were assembled by hand which surely has introduced some variations in the amount of glue and subsequent distance between the LC-tank and the aluminum target. Any excessive glue 
might have created unforeseen connections between the rubber medium and the LC-tank, which is likely to have created variations in drift and hysteresis. Still the differences between the sensors tested were fairly small. A more controlled production method will decrease these differences and can be expected to improve the reproducability of the performance of the sensors.

This sensor is very suitable for other biomedical applications where gradual changes of force should be measured in a small volume. Applications that come to mind are prosthetic socket fitting and prevention of pressure ulcers. For applications requiring a higher bandwidth, a sacrifice in power consumption should be made in the settings of the LDC1614. For applications requiring other force ranges, the stiffness of the medium may be changed.

\subsection{Limitations}

We did not have the means to perform the tests in an environment with a controlled temperature, barometric pressure, and humidity. Instead we used the fluctuations in temperature to determine its influence and correct the signal for it. This may have introduced a confirmation bias. Additionally, the temperature influence was determined with a load of $500 \mathrm{~g}$, and we did not test if this influence was load-dependent.

During the incremental load test, the maximum load $(1 \mathrm{~kg})$ was usually applied longer than the other loads. Due to the time drift, this might have lead to an overestimation of the calculated hysteresis.

\subsection{Sensor in Practice}

Preliminary results of measurements in the treatment of habitual toe walkers, Dupuytren fingers [21], and clubfeet [22] show excellent functioning for long-term measurement. Encountered problems in these applications were of a practical nature and were resolved with improved design: delamination of the aluminum target at the removal of the sensor was resolved by adding a post-it on top, battery failure was resolved by enhanced feedback with two LEDs on the AU and problems with identifying the start of important events in the logging was resolved by adding an input button on the AU.

\section{Conclusions}

A practical small-sized force sensor has been developed for long-term precision measurements in a portable setting. The used approach of inductive sensing combined with a compressible medium shows good applicability for the proposed force measurements between the skin and a cast, and great potential for many other applications.

Author Contributions: R.B.G. and V.I.S. conceived and designed the experiments; R.B.G. performed the experiments; R.B.G. analyzed the data; V.I.S. contributed materials/analysis tools; R.B.G. wrote the paper. V.I.S. and G.J.V. reviewed the paper.

Funding: This research was funded by [Stichting voor de Technische Wetenschappen] grant number [grant P12-03]. We received funds from the Open Science Fund from the University of Twente for covering the costs to publish in open access.

Acknowledgments: We would like to thank Jasper den Os for performing the drift measurements, Danny Bokma for the technical development of the electronics, Eline Kolk for helping with sensor manufacturing, Michel van Hirtum for working out the physics and Remco Wiegerink for his help in interpretation the data.

Conflicts of Interest: The authors declare no conflict of interest. The founding sponsors had no role in the design of the study; in the collection, analyses, or interpretation of data; in the writing of the manuscript; or in the decision to publish the results. 


\section{Abbreviations}

The following abbreviations are used in this manuscript:

$\begin{array}{ll}\text { OEM } & \text { Original Equipment Manufacturer } \\ \text { FSR } & \text { Force Sensitive Resistor } \\ \text { LDC } & \text { Inductance-to-Digital Converter } \\ \text { LC-tank } & \text { Inductor Capacitor tank } \\ \text { PCB } & \text { Printed Circuit Board } \\ \text { AU } & \text { Acquisition Unit (see Figure 2) } \\ \text { SLU } & \text { Sensing and Logging Unit (see Figure 2) }\end{array}$

\section{References}

1. Ponseti, I.V. Congenital Clubfoot: Fundamentals of Treatment, 2nd ed.; Oxford University Press Inc.: New York, NY, USA, 2008.

2. Hollinger, A.; Wanderley, M.M. Evaluation of Commercial Force-Sensing Resistors. In Proceedings of the International Conference on New Interfaces for Musical Expression (NIME’06), Paris, France, 4-8 June 2018; IRCAM: Paris, France, 2006.

3. Parmar, S.; Khodasevych, I.; Troynikov, O. Evaluation of Flexible Force Sensors for Pressure Monitoring in Treatment of Chronic Venous Disorders. Sensors 2017, 17, 1923, doi:10.3390/s17081923. [CrossRef] [PubMed]

4. Khodasevych, I.; Parmar, S.; Troynikov, O. Flexible Sensors for Pressure Therapy: Effect of Substrate Curvature and Stiffness on Sensor Performance. Sensors 2017, 17, 2399, doi:10.3390/s17102399. [CrossRef] [PubMed]

5. Texas Instruments. LDC1612, LDC1614 Multi-Channel 28-Bit Inductance to Digital Converter (LDC) for Inductive Sensing; Texas Instruments: Dallas, TX, USA, 2014.

6. Oberhauser, C. LDC Sensor Design; Application Report SNOA930; Texas Instruments: Dallas, TX, USA, 2015.

7. Kasemsadeh, B.; LaPointe, L. Inductive Sensing Touch-On-Metal Buttons Design Guide; Application Report SNOA951; Texas Instruments: Dallas, TX, USA, 2016.

8. Wang, H.; Kow, J.; de Boer, G.; Jones, D.; Alazmani, A.; Culmer, P. A low-cost, High-Performance, Soft Tri-axis Tactile Sensor based on Eddy-Current Effect. In Proceedings of the 2017 IEEE Sensors, Glasgow, UK, 29 October-1 November 2017; pp. 1-3, doi:10.1109/ICSENS.2017.8234098. [CrossRef]

9. Bonifas, A.P.; Kim, J.; Suh, J.-J. Force Responsive Inductors for Force Sensors. WO Patent PCT/US2015/ 017096, 3 September 2015.

10. Zinober, S.; Has, R. Wirbelstromsensor und Verfahren Zum Messen Einer Kraft. WO Patent PCT/EP2013/ 061024, 9 January 2014.

11. Maxim Integrated. DS1825 Programmable Resolution 1-Wire Digital Thermometer with 4-Bit ID; Maxim Integrated: San Jose, CA, USA, 2005.

12. NXP. Kinetis KL25 Sub-Family; NXP: Eindhoven, The Netherlands, 2014.

13. Holubeva, N. Configuring Inductive-to-Digital-Converters for Parallel Resistance (Rp) Variation in L-C Tank Sensors; Application Report SNAA221A; Texas Instruments: Dallas, TX, USA, 2015.

14. Giesberts, R.B. Mbed: Inductive_Sensor_3. Availeble online: https://os.mbed.com/users/bobgiesberts/ code/Inductive_Sensor_3 (accessed on 28 June 2018).

15. Riley, W. Handbook of Frequency: Stability Analysis; National Institute of Standards and Technology: Gaithersburg, MD ,USA, 2008.

16. Ferguson-Pell, M.; Hagisawa, S.; Bain, D. Evaluation of a sensor for low interface pressure applications. Med. Eng. Phys. 2000, 22, 657-663, doi:10.1016/S1350-4533(00)00080-1. [CrossRef]

17. Carr, J.J. Sensors and Circuits: Sensors, Transducers, and Supporting Circuits for Electronic Instrumentation, Measurement, and Control; PTR Prentice-Hall, Inc.: Englewood Cliffs, NJ, USA, 1993.

18. Oberhauser, C. Optimizing L Measurement Resolution for the LDC161x and LDC1101; Application Report SNOA944; Texas Instruments: Dallas, TX, USA, 2016.

19. Tekscan. Flexiforce ${ }^{\circledR}$ Sensors Users Manual; Tekscan: Boston, MA, USA, 2012.

20. Interlink Electronics. FSR ${ }^{\mathrm{TM}}$ Force Sensing Resistors ${ }^{\mathrm{TM}}$, FSR ${ }^{\mathrm{TM}}$ Integration Guide; Interlink Electronics: Camarillo, CA, USA, 2010. 
21. Giesberts, R.B.; ter Haar, A.M.; Sanderman, G.M.; Hekman, E.E.G.; Verkerke, G.J. Tissue adaptation rate in the treatment of Dupuytren contracture. J. Hand Ther. 2018, submitted.

22. Giesberts, R.B.; Hekman, E.E.G.; Verkerke, G.J.; Maathuis, P.G.M. Cast-Foot Interface Pressure in the Ponseti Method. Bone Jt. J. 2018, in preparation. 